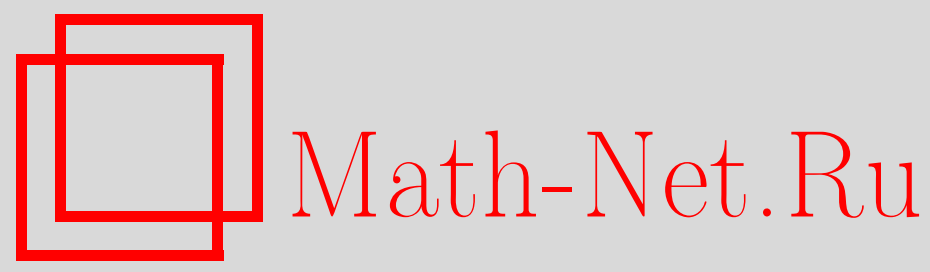

О. А. Логачев, С. В. Смышляев, В. В. Ященко, Новые методы изучения совершенно уравновешенных булевых функций, Дискрет. матем., 2009, том 21, выпуск 2, 51-74

DOI: https://doi.org/10.4213/dm1046

Использование Общероссийского математического портала Math-Net.Ru подразумевает, что вы прочитали и согласны с пользовательским соглашением http://www . mathnet.ru/rus/agreement

Параметры загрузки:

IP: 54.210 .77 .194

26 апреля 2023 г., $11: 17: 43$ 
УДК 519.7

\title{
Новые методы изучения совершенно уравновешенных булевых функций
}

\author{
() 2009 г. О. А. Логачев, С. В. Смышляев, В. В. Ященко
}

В работе рассмотрены новые подходы к изучению и введены параметры для описания некоторых новых классов совершенно уравновешенных булевых функций.

Работа первого и третьего авторов поддержана Российским фондом фундаментальных исследований, проект 07-0100154.

\section{1. Введение}

Одним из важных классов преобразований двоичных последовательностей являются преобразования, осуществляемые с помощью регистра сдвига и булевой функции. В технике связи такое дискретное устройство называется кодирующим устройством с конечной памятью (см. [1, 2]), а в криптографии - фильтрующим генератором (см. [3, 4]).

С криптографической точки зрения важными для данного дискретного устройства являются исследования статистических свойств выходных последовательностей в плане близости их к свойствам случайных последовательностей (в рамках соответствующих теоретико-вероятностных моделей) и возможности однозначного восстановления входной последовательности по выходной последовательности, начальному и конечному состояниям.

Как оказалось, оба этих вопроса тесно связаны со свойством булевой функции, которое мы будем называть совершенной уравновешенностью. В настоящей работе мы используем для вводимых в определениях 1, 2 функций несколько иные термины, нежели в [5]. Они представляются нам более формализованными и не содержат элементов, требующих дополнительной интерпретации. Длительное время считалось, что этим свойством обладают лишь линейные по первой или последней переменной функции. В работе [5] была опровергнута эта гипотеза и приведены примеры функций, обладающих свойством совершенной уравновешенности и не являющихся линейными ни по одной из крайних переменных. В этой же работе показана эквивалентность совершенной уравновешенности булевой функции и сюръективности соответствующего автоматного отображения, а также исследованы некоторые свойства указанных отображений, не являющихся сюръективными. В работах $[6,7]$, в частности, обсуждаются криптографические аспекты использования совершенно уравновешенных функций в качестве фильтрующих функций. Необходимо также указать на ошибочность результата, связанного со статистическими свойствами выходных последовательностей фильтрующих генераторов, полученного в работе [8] без использования адекватной теоретико-вероятностной модели.

В настоящей работе мы вводим некоторые новые понятия, связанные со свойством совершенной уравновешенности, приводим новые критерии этого свойства, описываем 


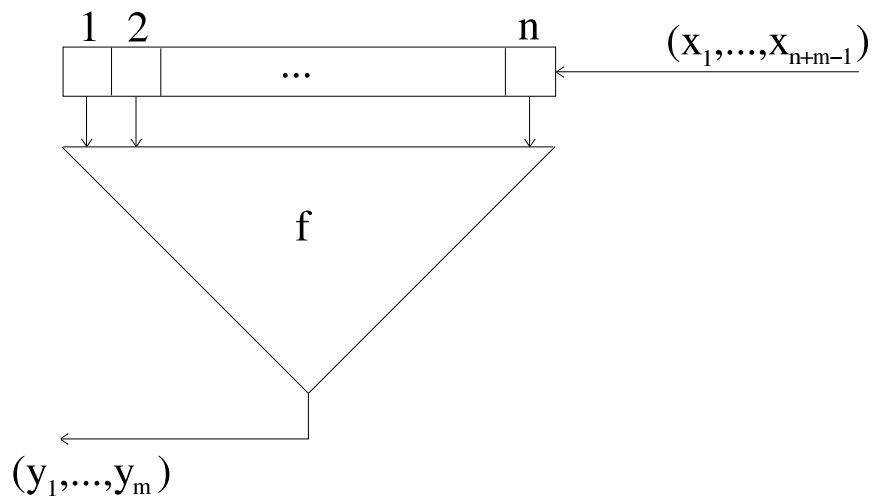

Рис. 1. Схема функционирования кодирующего устройства

некоторые классы совершенно уравновешенных булевых функций, а также приводим результаты экспериментальных исследований.

Работа организована следующим образом. В разделе 2 приводятся основные используемые нами определения и обозначения. Раздел 3 содержит необходимые для изложения предварительные результаты. В разделе 4 приводятся формулировки и доказательства основных результатов, а также результаты экспериментальных исследований. В разделе 5 намечены некоторые направления дальнейших исследований.

\section{2. Основные определения и обозначения}

Пусть $\mathbf{F}_{2}-$ поле Галуа из двух элементов, $\mathrm{V}_{n}=\mathbf{F}_{2}^{n}-$ пространство наборов длины $n \in \mathbf{N}$ над полем $\mathbf{F}_{2}$. Будем обозначать через $\mathscr{F}_{n}$ множество булевых функций от $n$ переменных $\left\{x_{1}, \ldots, x_{n}\right\}$. Крайними переменными функции $f \in \mathscr{F}_{n}$ будем называть $x_{1}$ и $x_{n}$. Через $\Phi_{n}$ будем обозначать подмножество функций из $\mathscr{F}_{n}$, существенно зависящих от обеих крайних переменных. Пусть $m \in \mathbf{N}$. Рассмотрим систему булевых уравнений

$$
f\left(x_{s}, \ldots, x_{s+n-1}\right)=y_{s}, \quad s=1, \ldots, m .
$$

Легко видеть, что система (1) описывает функционирование (в смысле зависимости выходных символов от входных) в течение $m$ тактов кодирующего устройства (см. [1]), построенного с помощью регистра сдвига с $n$ ячейками двоичной памяти и булевой функции $f$ (см. рис. 1).

Для $f \in \mathscr{F}_{n}$ обозначим через $f_{m}$ отображение из $\mathrm{V}_{m+n-1}$ в $\mathrm{V}_{m}$ вида

$$
f_{m}\left(x_{1}, \ldots, x_{m+n-1}\right)=\left(f\left(x_{1}, \ldots, x_{n}\right), f\left(x_{2}, \ldots, x_{n+1}\right), \ldots, f\left(x_{m}, \ldots, x_{m+n-1}\right)\right) .
$$

Для булевой функции $f$ введем понятие дефекта $\operatorname{Def}_{m}(f)$, определив для произвольного $m \in \mathbf{N}$ множество

$$
J(f, m)=\mathrm{V}_{m} \backslash \operatorname{Im}\left(f_{m}\right)
$$

и положив

$$
\operatorname{Def}_{m}(f)=|J(f, m)|,
$$

где $|M|$ - мощность множества $M$. В работе [5] элементы множества $J(f, m)$ называются запретами функции $f$ длины $m$. 
Определение 1 ([5]). Булева функция $f \in \mathscr{F}_{n}$ называется функцией дефекта нуль, если $\operatorname{Def}_{m}(f)=0$ для любого $m \in \mathbf{N}$.

Легко видеть (см. [5]), что если функция линейна хотя бы по одной из крайних переменных, то она является функцией дефекта нуль. Обозначим $\mathscr{L}_{n}$ множество функций из $\mathscr{F}_{n}$, линейных по первой переменной, а $\mathscr{R}_{n}$ множество функций из $\mathscr{F}_{n}$, линейных по последней переменной.

Определение 2 ([5]). Булева функция $f \in \mathscr{F}_{n}$ называется совершенно уравновешенной, если отображение $f_{m}: \mathrm{V}_{m+n-1} \rightarrow \mathrm{V}_{m}$ является уравновешенным (см. [3], с. 104) для любого $m \in \mathbf{N}$, то есть соотношение $\left|\left(f_{m}\right)^{-1}(y)\right|=2^{n-1}$ выполняется для любого $m \in \mathbf{N}$ и любого $y \in \mathrm{V}_{m}$.

Из определения 2 при $m=1$ нетрудно видеть, что совершенно уравновешенная функция является уравновешенной (то есть вес $f$ равен $2^{n-1}$ ). Следовательно, относительная доля совершенно уравновешенных булевых функций в $\mathscr{F}_{n}$ стремится к нулю при $n \rightarrow \infty$.

\section{3. Предварительные результаты}

Теорема 1 ([5]). Булева функиия $f \in \mathscr{F}_{n}$ является функичей дефекта нуль тогда и только тогда, когда она совершенно уравновешена.

Множество совершенно уравновешенных функций (функций дефекта нуль) из $\mathscr{F}_{n}$ обозначим $\mathscr{P} \mathscr{B}_{n}$. Особый интерес представляют функции из $\Phi_{n} \cap \mathscr{P}_{\mathscr{B}_{n}} \backslash\left(\mathscr{L}_{n} \cup \mathscr{R}_{n}\right)$.

Необходимо также отметить преобразования множества $\mathscr{F}_{n}$, оставляющие инвариантным множество $\mathscr{P} \mathscr{B}_{n}$ (см. [5]):

(1) $\gamma_{0}: f\left(x_{1}, \ldots, x_{n}\right) \rightarrow f\left(x_{1}, \ldots, x_{n}\right) \oplus 1$,

(2) $\gamma_{1}: f\left(x_{1}, \ldots, x_{n}\right) \rightarrow f\left(x_{1} \oplus 1, \ldots, x_{n} \oplus 1\right)$,

(3) $\gamma_{2}: f\left(x_{1}, \ldots, x_{n}\right) \rightarrow f\left(x_{n}, \ldots, x_{1}\right)$.

Определенный интерес представляют условия равномерности распределения правых частей системы уравнений (1) при условии равномерности распределения случайного вектора $X_{m}=\left(x_{1}, \ldots, x_{m+n-1}\right)$.

Теорема 2 ([3]). Пусть $n \in \mathbf{N} u f \in \mathscr{F}_{n}$. Пусть $\left\{X_{m}=\left(x_{1}, \ldots, x_{m+n-1}\right)\right\}_{m=1}^{\infty}-$ последовательность случайных векторов с распределением

$$
\mathbf{P}\left\{X_{m}=\left(a_{1}, \ldots, a_{m+n-1}\right)\right\}=2^{-(m+n-1)}
$$

для любых $\left(a_{1}, \ldots, a_{m+n-1}\right) \in \mathrm{V}_{m+n-1}$. Случайный вектор $Y_{m}=f_{m}\left(X_{m}\right)$ распределен равномерно для любого $m \in \mathbf{N}$ тогда и только тогда, когда $f$-совершенно уравновешенная функиия.

Теорема 3 ([5]). Булева функция $f \in \mathscr{F}_{n}$ является совершенно уравновешенной тогда $u$ только тогда, когда не существует двух двоичных последовательностей

$$
x=\left(x_{1}, \ldots, x_{r}\right), \quad z=\left(z_{1}, \ldots, z_{r}\right) \in \mathrm{V}_{r}, \quad r>2 n,
$$

таких, что

$$
\begin{aligned}
x_{1}=z_{1}, \ldots, x_{n} & =z_{n}, x_{r-n+1}=z_{r-n+1}, \ldots, x_{r}=z_{r} \\
x & \neq z ; \\
f\left(x_{i}, \ldots, x_{i+n-1}\right) & =f\left(z_{i}, \ldots, z_{i+n-1}\right), \quad i=1, \ldots, r-n+1 .
\end{aligned}
$$




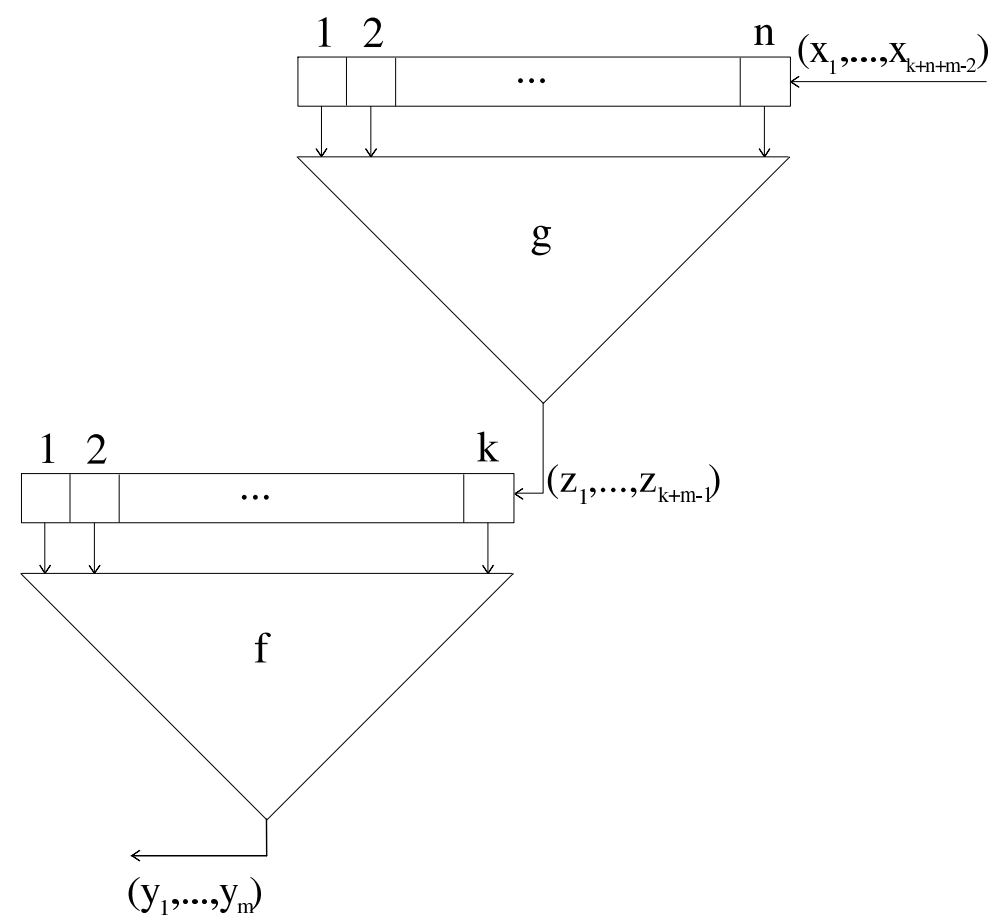

Рис. 2. Схема функционирования кодирующего устройства с функцией $f[g]$

Для пары натуральных чисел $k, n$ рассмотрим отображение $\Xi_{k, n}$ из $\mathscr{F}_{k} \times \mathscr{F}_{n}$ в $\mathscr{F}_{k+n-1}$ вида

$$
\Xi_{k, n}(f, g)=f[g]=h \in \mathscr{F}_{k+n-1}, \quad f \in \mathscr{F}_{k}, \quad g \in \mathscr{F}_{n},
$$

где

$$
\begin{aligned}
h\left(x_{1}, \ldots, x_{k+n-1}\right) & =f[g]\left(x_{1}, \ldots, x_{k+n-1}\right) \\
& =f\left(g\left(x_{1}, \ldots, x_{n}\right), g\left(x_{2}, \ldots, x_{n+1}\right), \ldots, g\left(x_{k}, \ldots, x_{k+n-1}\right)\right) .
\end{aligned}
$$

Отображение $\Xi_{k, n}$ задает композицию кодирующих устройств, изображенную на рис. 2 . Для этой конструкции справедливо следующее утверждение.

Теорема 4 ([9]). Пусть $f \in \mathscr{F}_{k}, g \in \mathscr{F}_{n}$. Функция $f[g] \in \mathscr{F}_{k+n-1}$ совершенно уравновешена тогда и только тогда, когда функции $f$ и $g$ совершенно уравновешень.

Теорема 4 означает, что множество совершенно уравновешенных булевых функций замкнуто относительно введенной операции композиции. Поэтому, в частности, зная совершенно уравновешенные функции от меньшего числа переменных, можно построить достаточно много совершенно уравновешенных функций от большего числа переменных. На этом пути легко также построить достаточно много совершенно уравновешенных функций из $\Phi_{n} \cap \mathscr{P}_{n} \backslash\left(\mathscr{L}_{n} \cup \mathscr{R}_{n}\right)$.

Лемма 1. Пусть $f \in \Phi_{k} \cap \mathscr{P} \mathscr{B}_{k} \backslash\left(\mathscr{L}_{k} \cup \mathscr{R}_{k}\right), g \in \Phi_{n} \cap \mathscr{P}_{\mathscr{B}_{n}} \backslash\left(\mathscr{L}_{n} \cup \mathscr{R}_{n}\right)$. Тогда

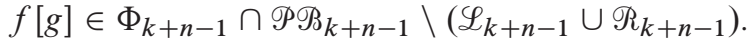




\section{4. Основные результаты}

Поскольку свойство уравновешенности булевых отображений является сводимым (см. с. 112 в [3]), из теоремы 2.139 в [3] и определения 2 вытекает следующее утверждение.

Лемма 2. Булева функция $f \in \mathscr{F}_{n}$ является совершенно уравновешенной тогда и только тогда, когда для любого $m \in \mathbf{N} u$ любого ненулевого $b=\left(b_{1}, \ldots, b_{m}\right) \in \mathrm{V}_{m}$ булева функичия

$$
\begin{aligned}
f_{b}\left(x_{1}, \ldots, x_{m+n-1}\right)=b_{1} f\left(x_{1}, \ldots, x_{n}\right) \oplus b_{2} f\left(x_{2}, \ldots, x_{n+1}\right) & \oplus \ldots \\
& \oplus b_{m} f\left(x_{m}, \ldots, x_{m+n-1}\right)
\end{aligned}
$$

является уравновешенной.

Условимся о следующем правиле: для любой функции $\varphi \in \Phi_{n}$ через $\varphi_{0}, \varphi_{1} \in \mathscr{F}_{n-1}$ будем обозначать коэффициенты разложения $\varphi$ по правой крайней переменной, то есть,

$$
\varphi\left(x_{1}, \ldots, x_{n-1}, x_{n}\right)=\varphi_{0}\left(x_{1}, \ldots, x_{n-1}\right) \oplus \varphi_{1}\left(x_{1}, \ldots, x_{n-1}\right) x_{n} .
$$

Разложение можно продолжить, применяя и дальше это правило:

$$
\begin{array}{r}
\varphi\left(x_{1}, \ldots, x_{n-1}, x_{n}\right)=\varphi_{00}\left(x_{1}, \ldots, x_{n-2}\right) \oplus \varphi_{01}\left(x_{1}, \ldots, x_{n-2}\right) x_{n-1} \oplus \\
\oplus \varphi_{10}\left(x_{1}, \ldots, x_{n-2}\right) x_{n} \oplus \varphi_{11}\left(x_{1}, \ldots, x_{n-2}\right) x_{n-1} x_{n} .
\end{array}
$$

Для булевой функции $f \in \mathscr{F}_{n}$ определим конечный инициальный автомат Мура (см. [10]) $Z_{f}=\left(2^{\mathrm{V}_{n-1}}, \mathbf{F}_{2}, \lambda, \mathrm{V}_{n-1}\right)$, у которого:

- множество состояний совпадает с множеством всех подмножеств векторного пространства $\mathrm{V}_{n-1}$;

- входным алфавитом является $\mathbf{F}_{2}$;

- начальным состоянием является $\mathrm{V}_{n-1}$;

- функция переходов $\lambda: 2^{\mathrm{V}_{n-1}} \times \mathbf{F}_{2} \rightarrow 2^{\mathrm{V}_{n-1}}$ задается следующими правилами:

$$
\lambda\left(s_{1} \cup s_{2}, y\right)=\lambda\left(s_{1}, y\right) \cup \lambda\left(s_{2}, y\right)
$$

для любых $s_{1}, s_{2} \in 2^{\mathrm{V}_{n-1}}, y \in \mathbf{F}_{2}$,

$$
\lambda\left(\left(x_{1}, \ldots, x_{n-1}\right), y\right)=\left\{\left(x_{2}, \ldots, x_{n-1}, 0\right),\left(x_{2}, \ldots, x_{n-1}, 1\right)\right\},
$$

если $f_{1}\left(x_{1}, \ldots, x_{n-1}\right)=0, f_{0}\left(x_{1}, \ldots, x_{n-1}\right)=y$;

$$
\lambda\left(\left(x_{1}, \ldots, x_{n-1}\right), y\right)=\varnothing,
$$

если $f_{1}\left(x_{1}, \ldots, x_{n-1}\right)=0, f_{0}\left(x_{1}, \ldots, x_{n-1}\right)=y \oplus 1$;

$$
\lambda\left(\left(x_{1}, \ldots, x_{n-1}\right), y\right)=\left\{\left(x_{2}, \ldots, x_{n-1}, f_{0}\left(x_{1}, \ldots, x_{n-1}\right) \oplus y\right)\right\},
$$

если $f_{1}\left(x_{1}, \ldots, x_{n-1}\right)=1$. 
Очевидно, что автомат $Z_{f}$ моделирует процесс построения множества решений системы (1) путем последовательного продолжения решений первого уравнения до решений двух первых уравнений и так далее. Поэтому в поведении автомата $Z_{f}$ содержится, в частности, информация о множестве запретов функции $f$, что отражено в теореме 5 , доказательство которой достаточно очевидно.

Теорема 5. Пусть под действием входного слова $y_{1}, \ldots, y_{m}$ автомат $Z_{f}$ переходит в состояние $s \in 2^{\mathrm{V}_{n-1}}$, а множеством решений системы (1) является множество $M_{f} \subseteq \mathrm{V}_{n+m-1}$. Тогда

(1) множество s состоит из тех и только тех $\left(x_{m+1}, \ldots, x_{n+m-1}\right) \in \mathrm{V}_{n-1}$, которье являются подвекторами (на соответствующих координатах) векторов из $M_{f}$;

(2) множеством запретов функиии $f$ являются те и только те слова $y_{1}, \ldots, y_{m}$, под действием которых автомат $Z_{f}$ переходит в состояние $\varnothing \in 2^{\mathrm{V}_{n-1}}$;

(3) функция $f$ является совершенно уравновешенной тогда и только тогда, когда автомат $Z_{f}$ не может быть переведен в состояние $\varnothing \in 2^{\mathrm{V}_{n-1}}$.

В качестве примера использования теоремы 5 рассмотрим простейшую функцию, которая нелинейно зависит от $x_{n}$. Пусть $f_{1}\left(x_{1}, \ldots, x_{n-1}\right)=0$ тогда и только тогда, когда $\left(x_{1}, \ldots, x_{n-1}\right)=\varepsilon \in \mathrm{V}_{n-1}$. Легко видеть, что в этом случае мощности множеств $s, \lambda(s, y)$ связаны соотношениями

$$
|\lambda(s, y)|= \begin{cases}|s|, & \text { если } \varepsilon \notin s, \\ |s|-1, & \text { если } \varepsilon \in s, \quad y=f_{0}(\varepsilon) \oplus 1, \\ |s|+1, & \text { если } \varepsilon \in s, \quad y=f_{0}(\varepsilon) .\end{cases}
$$

Теперь уже простыми рассуждениями с использованием математической индукции можно доказать следующее утверждение.

Предложение 1. Минимальная длина запрета функиии $f \in \mathscr{F}_{n}$, для которой соотношение $f_{1}\left(x_{1}, \ldots, x_{n-1}\right)=0$ выполняется только на одном векторе из $\mathrm{V}_{n-1}$, равна $2^{n-1}$.

Теорема 6. Пусть $f \in \mathscr{F}_{n}$, и в условиях теоремь 2 пусть

$$
i_{m}(f)=m^{-1} I\left(Y_{m}, X_{m}\right),
$$

где $I\left(Y_{m}, X_{m}\right)$ - средняя взаимная информация между случайными векторами $Y_{m} u X_{m}$. Тогда справедливы следующие утверждения:

(1) функция $f$ является совершенно уравновешенной, если и только если $i_{m}(f)=1$ для любого натурального $\mathrm{m}$;

(2) для произвольной функции $g \in \mathscr{F}_{n}$ справедливо неравенство $i_{m}(g) \leqslant 1$.

Доказательство. Пусть $f \in \mathscr{F}_{n}$. В условиях теоретико-вероятностных предположений теоремы 2 выпишем выражения для следующих вероятностей:

$$
\begin{aligned}
p_{a} & =\mathbf{P}\left\{X_{m}=a\right\}=2^{-(m+n-1)}, \quad a \in \mathrm{V}_{m+n-1}, \\
p_{b / a} & =\mathbf{P}\left\{Y_{m}=b \mid X_{m}=a\right\}= \begin{cases}1, & \text { если } a \in f_{m}^{-1}(b), \\
0, & \text { если } a \notin f_{m}^{-1}(b),\end{cases} \\
p_{b, a} & =\mathbf{P}\left\{Y_{m}=b, X_{m}=a\right\}=p_{a} p_{b / a}= \begin{cases}2^{-(m+n-1)}, & \text { если } a \in f_{m}^{-1}(b), \\
0, & \text { если } a \notin f_{m}^{-1}(b),\end{cases} \\
q_{b} & =\mathbf{P}\left\{Y_{m}=b\right\}=2^{-(m+n-1)}\left|f_{m}^{-1}(b)\right|, \quad b \in \mathrm{V}_{m} .
\end{aligned}
$$


Тогда, учитывая равенство $0 \log 0=0$, получаем, что

$$
\begin{aligned}
i_{m}(f) & =m^{-1} I\left(Y_{m}, X_{m}\right)=m^{-1} \sum_{b \in \mathrm{V}_{m}} \sum_{a \in \mathrm{V}_{m+n-1}} p_{b, a} \log _{2} \frac{p_{b / a}}{q_{b}} \\
& =m^{-1} \sum_{b \in \mathrm{V}_{m}} \sum_{a \in f_{m}^{-1}(b)} p_{b, a} \log _{2} \frac{p_{b / a}}{q_{b}} \\
& =m^{-1} \sum_{b \in \mathrm{V}_{m}} \sum_{a \in f_{m}^{-1}(b)} 2^{-(m+n-1)}\left((m+n-1)-\log _{2}\left|f_{m}^{-1}(b)\right|\right) \\
& =m^{-1} \sum_{b \in \mathrm{V}_{m}}\left|f_{m}^{-1}(b)\right| 2^{-(m+n-1)}\left((m+n-1)-\log _{2}\left|f_{m}^{-1}(b)\right|\right) \\
& =m^{-1}(m+n-1)-2^{-(m+n-1)} \sum_{b \in \mathrm{V}_{m}}\left|f_{m}^{-1}(b)\right| \log _{2}\left|f_{m}^{-1}(b)\right| .
\end{aligned}
$$

Докажем первое утверждение. Пусть $f \in \mathscr{P}_{\mathscr{P}_{n}}$. Тогда

$$
\left|f_{m}^{-1}(b)\right|=2^{n-1}
$$

для любого $b \in \mathrm{V}_{m}$ и для любого натурального $m$. Следовательно, $i_{m}(f)=1$ для любого $m \in \mathbf{N}$.

Обратно, пусть $f \in \mathscr{F}_{n}$ и $i_{m}(f)=1$ для любого натурального $m$. Тогда, воспользовавшись полученным выше соотношением, получаем, что

$$
2^{-(m+n-1)} \sum_{b \in \mathrm{V}_{m}}\left|f_{m}^{-1}(b)\right| \log _{2}\left|f_{m}^{-1}(b)\right|=n-1 .
$$

Поскольку

$$
\sum_{b \in \mathrm{V}_{m}}\left|f_{m}^{-1}(b)\right| / 2^{m+n-1}=1,
$$

с учетом последнего соотношения легко получить, что

$$
\sum_{b \in \mathrm{V}_{m}} \frac{\left|f_{m}^{-1}(b)\right|}{2^{m+n-1}} \log _{2} \frac{\left|f_{m}^{-1}(b)\right|}{2^{m+n-1}}=\sum_{b \in \mathrm{V}_{m}} 2^{-m} \log _{2} \frac{2^{n-1}}{2^{m+n-1}}=\sum_{b \in \mathrm{V}_{m}} 2^{-m} \log _{2} \frac{1}{2^{m}}
$$

В силу свойств энтропии (см. [11]) это возможно тогда и только тогда, когда

$$
\left|f_{m}^{-1}(b)\right| / 2^{m+n-1}=2^{-m}
$$

при любом $b \in \mathrm{V}_{m}$, то есть, при $\left|f_{m}^{-1}(b)\right|=2^{n-1}$. Следовательно, $f \in \mathscr{P}_{\mathscr{B}_{n}}$.

Докажем второе утверждение. Пусть $g$ - произвольная булева функция из $\mathscr{F}_{n}$ и $f \in \mathscr{P}_{n}$. Тогда

$$
\begin{aligned}
i_{m}(f)-i_{m}(g) & =m^{-1}\left(2^{-(m+n-1)} \sum_{b \in \mathrm{V}_{m}}\left|g_{m}^{-1}(b)\right| \log _{2}\left|g_{m}^{-1}(b)\right|-(n-1)\right) \\
& =m^{-1}\left(\sum_{b \in \mathrm{V}_{m}} \frac{\left|g_{m}^{-1}(b)\right|}{2^{m+n-1}} \log _{2}\left|g_{m}^{-1}(b)\right|-\sum_{b \in \mathrm{V}_{m}} \frac{\left|g_{m}^{-1}(b)\right|}{2^{m+n-1}} \log _{2} 2^{n-1}\right) \\
& =m^{-1}\left(\sum_{b \in \mathrm{V}_{m}} \frac{\left|g_{m}^{-1}(b)\right|}{2^{m+n-1}} \log _{2}\left(\frac{\left|g_{m}^{-1}(b)\right|}{2^{m+n-1}} / \frac{2^{n-1}}{2^{m+n-1}}\right)\right) .
\end{aligned}
$$


Как мы уже отмечали ранее,

$$
\sum_{b \in \mathrm{V}_{m}}\left|g_{m}^{-1}(b)\right| / 2^{m+n-1}=1,
$$

поэтому можно воспользоваться неравенством

$$
\sum_{j=1}^{s} \alpha_{j} \log \alpha_{j} \geqslant \sum_{j=1}^{s} \alpha_{j} \log \beta_{j}
$$

где

$$
\sum_{j=1}^{s} \alpha_{j}=\sum_{j=1}^{s} \beta_{j}=1, \quad \alpha_{j} \geqslant 0, \quad \beta_{j} \geqslant 0,
$$

применяя которое, получаем, что

$$
i_{m}(f)-i_{m}(g)=1-i_{m}(g) \geqslant 0 .
$$

Таким образом, на совершенно уравновешенных функциях и только на них значение функционала $i_{m}(f)$ достигает максимума. Другими словами, в среднем в одном бите выходной последовательности устройства (см. рис. 1) с фильтрующей совершенно уравновешенной функцией содержится информации (о входной последовательности) не меньше, чем у аналогичного устройства с произвольной фильтрующей функцией.

Определение 3. Графом сдвигов функции $f \in \mathscr{F}_{n}$ назовем ориентированный граф $\Gamma_{f}=(G, V),|G|=2^{2 n-3}+2^{n-2}$ (без кратных ребер, с петлями), каждая вершина которого поставлена во взаимно однозначное соответствие неупорядоченной паре двоичных векторов длины $n-1$, а ребро из вершины $\left(\begin{array}{c}\left(x_{1}, \ldots, x_{n-1}\right) \\ \left(z_{1}, \ldots, z_{n-1}\right)\end{array}\right)$ в вершину $\left(\begin{array}{c}\left(u_{1}, \ldots, u_{n-1}\right) \\ \left(v_{1}, \ldots, v_{n-1}\right)\end{array}\right)$ присутствует в графе тогда и только тогда, когда выполнено по крайней мере одно из двух условий,

$$
\begin{aligned}
\left(x_{2}, \ldots, x_{n-1}\right) & =\left(u_{1}, \ldots, u_{n-2}\right), \\
\left(z_{2}, \ldots, z_{n-1}\right) & =\left(v_{1}, \ldots, v_{n-2}\right), \\
f\left(x_{1}, \ldots, x_{n-1}, u_{n-1}\right) & =f\left(z_{1}, \ldots, z_{n-1}, v_{n-1}\right)
\end{aligned}
$$

или

$$
\begin{aligned}
\left(x_{2}, \ldots, x_{n-1}\right) & =\left(v_{1}, \ldots, v_{n-2}\right), \\
\left(z_{2}, \ldots, z_{n-1}\right) & =\left(u_{1}, \ldots, u_{n-2}\right), \\
f\left(x_{1}, \ldots, x_{n-1}, v_{n-1}\right) & =f\left(z_{1}, \ldots, z_{n-1}, u_{n-1}\right) .
\end{aligned}
$$

Через $A_{f} \subset \Gamma_{f}$ обозначим подграф графа сдвигов, отвечающий множеству пар равных векторов длины $n-1$, через $\Gamma_{f}^{*}-$ граф, полученный из графа $\Gamma_{f}$ удалением всех ребер, лежащих внутри $A_{f}$. Каждому ориентированному пути в графе $\Gamma_{f}$ естественным образом соответствует пара двоичных последовательностей, составленных из меток вершин.

Теорема 7. Функиия $f \in \mathscr{F}_{n}$ является совершенно уравновешенной тогда и только тогда, когда в графе $\Gamma_{f}^{*}$ не существует ориентированного пути с началом и кониом $в$ nодграфе $A_{f}$. 
Доказательство. Из определения 3 видно, что упомянутому пути соответствует пара двоичных последовательностей $x, z$, удовлетворяющая условиям (5)-(7). Отсюда и из теоремы 3 получаем требуемое утверждение.

Рассмотрим множество $\Pi_{f}$ всех ориентированных путей в графе $\Gamma_{f}^{*}$ с началом в подграфе $A_{f}$. Очевидно, что принципиально возможны три ситуации:

(1) в множестве $\Pi_{f}$ найдется путь, заканчивающийся в подграфе $A_{f}$;

(2) ни один путь из множества $\Pi_{f}$ не оканчивается в $A_{f}$, и длина всех путей в $\Pi_{f}$ ограничена;

(3) ни один путь из множества $\Pi_{f}$ не оканчивается в $A_{f}$, и в $\Pi_{f}$ существует путь бесконечной длины.

В соответствии с теоремой 7 ситуация 1 не соответствует совершенно уравновешенным функциям, а ситуации 2 и 3 соответствуют совершенно уравновешенным функциям. Разбиение совершенно уравновешенных функций на два класса позволяет находить новые конструктивные критерии и параметры для их изучения.

Класс совершенно уравновешенных функций, соответствующих ситуации 2, можно изучать, последовательно увеличивая границу длины всех путей в $\Pi_{f}$.

Определение 4. Булева функция $f \in \mathscr{F}_{n}$ называется функцией с правым барьером длины $k \in \mathbf{N}$, если система уравнений

$$
\begin{aligned}
f\left(y_{1}, y_{2}, \ldots, y_{n}\right) & =f\left(z_{1}, z_{2}, \ldots, z_{n}\right), \\
f\left(y_{2}, y_{3}, \ldots, y_{n+1}\right) & =f\left(z_{2}, z_{3}, \ldots, z_{n+1}\right), \\
& \ldots \\
f\left(y_{k-1}, y_{k}, \ldots, y_{k+n-2}\right) & =f\left(z_{k-1}, z_{k}, \ldots, z_{k+n-2}\right), \\
y_{1}=z_{1}=x_{1}, \ldots, y_{n-1} & =z_{n-1}=x_{n-1}, y_{n}=0, z_{n}=1
\end{aligned}
$$

имеет решение, а система

$$
\begin{aligned}
f\left(y_{1}, y_{2}, \ldots, y_{n}\right) & =f\left(z_{1}, z_{2}, \ldots, z_{n}\right), \\
f\left(y_{2}, y_{3}, \ldots, y_{n+1}\right) & =f\left(z_{2}, z_{3}, \ldots, z_{n+1}\right) \\
& \ldots \\
f\left(y_{k-1}, y_{k}, \ldots, y_{k+n-2}\right) & =f\left(z_{k-1}, z_{k}, \ldots, z_{k+n-2}\right), \\
f\left(y_{k}, y_{k+1}, \ldots, y_{k+n-1}\right) & =f\left(z_{k}, z_{k+1}, \ldots, z_{k+n-1}\right), \\
y_{1}=z_{1}=x_{1}, \ldots, y_{n-1} & =z_{n-1}=x_{n-1}, y_{n}=0, z_{n}=1
\end{aligned}
$$

решений не имеет.

Определение 5. Булева функция $f \in \mathscr{F}_{n}$ называется функцией с левым барьером длины $k \in \mathbf{N}$, если $f\left(x_{n}, \ldots, x_{1}\right)$ является функцией с правым барьером длины $k$.

Замечание 1. Легко заметить, что преобразования $\gamma_{0}, \gamma_{1}$ сохраняют множества функций с правым и левым барьерами, преобразование $\gamma_{2}$ меняет множества функций с правым и левым барьерами местами, а также сохраняет множество совершенно уравновешенных функций без барьера. 
В соответствии с замечанием 1, достаточно ограничиться изучением функций с правым барьером.

Определение 6. Булева функция $f \in \mathscr{F}_{n}$ имеет барьер, если она имеет правый или левый барьер, или оба сразу. При этом длиной барьера функции называется соответственно длина правого барьера, левого барьера, или меньшая из длин барьеров.

Замечание 2. Из определений 4, 5 следует, что если $f\left(x_{1}, \ldots, x_{n}\right)$ имеет правый (левый) барьер длины $k$,

$$
\begin{aligned}
& g\left(x_{1}, \ldots, x_{n+m}\right) \equiv f\left(x_{1}, \ldots, x_{n}\right), \\
& h\left(x_{1}, \ldots, x_{n+m}\right) \equiv f\left(x_{m+1}, \ldots, x_{n+m}\right),
\end{aligned}
$$

то $g$ имеет правый барьер длины $k+m$ (левый барьер длины $k$ ), $h$ имеет правый барьер длины $k$ (левый барьер длины $k+m$ ).

Легко доказать следующее утверждение.

Предложение 2. Булева функиия имеет правый (левый) барьер длины $k=1$ тогда $u$ только тогда, когда она лежит в классе $\mathscr{R}_{n}$ (соответственно $\mathscr{L}_{n}$ ).

Приведем пример совершенно уравновешенной функции, соответствующей ситуации 3.

Предложение 3. Булева функиия $f\left(x_{1}, x_{2}, x_{3}, x_{4}, x_{5}\right)$, равная $x_{1} \oplus x_{2} \oplus x_{5} \oplus x_{1} x_{2} \oplus x_{1} x_{3} \oplus$ $x_{1} x_{4} \oplus x_{2} x_{3} \oplus x_{2} x_{4} \oplus x_{3} x_{4} \oplus x_{4} x_{5} \oplus x_{1} x_{3} x_{4} \oplus x_{2} x_{3} x_{4}$, является совершенно уравновешенной функиией без барьера.

Доказательство. Заметим, что $f$ можно представить с помощью конструкции (8) в таком виде:

$$
f\left(x_{1}, x_{2}, x_{3}, x_{4}, x_{5}\right)=g^{\gamma_{2}}[g],
$$

где

$$
g\left(x_{1}, x_{2}, x_{3}\right)=x_{1} \oplus x_{2} \oplus x_{3} \oplus x_{2} x_{3}, \quad g^{\gamma_{2}}\left(x_{1}, x_{2}, x_{3}\right) \equiv g\left(x_{3}, x_{2}, x_{1}\right) .
$$

Функция $g$ имеет левый барьер длины $k=1$, следовательно, $g, g^{\gamma_{2}} \in \mathscr{P}_{\mathscr{B}_{3}}$. Используя теорему 4 , получим, что $f \in \mathscr{P}_{5}$.

Чтобы доказать отсутствие барьеров у функции $f$, рассмотрим две пары последовательностей

$$
\begin{gathered}
\left(\begin{array}{l}
0,0,0,1,(0,1,0,1,0,0,)(0,1,0,1,0,0,)(0,1,0,1,0,0,) \ldots \\
0,0,0,1,(1,0,0,0,1,0,)(1,0,0,0,1,0,)(1,0,0,0,1,0,) \ldots
\end{array}\right), \\
\left(\begin{array}{l}
\ldots(0,0,1,1,1,1,1,0,)(0,0,1,1,1,1,1,0,)(0,0,1,1,1,1,1,0,) 0,0,0,1,0,0 \\
\ldots(1,1,1,0,0,0,1,1,)(1,1,1,0,0,0,1,1,)(1,1,1,0,0,0,1,1,) 0,1,0,1,0,0
\end{array}\right) .
\end{gathered}
$$

Для любого наперед заданного $k>0$ продолжим пару (12) до длины, большей $k+3$, воспользовавшись ее периодичностью. Затем, подставив ее в системы уравнений (10) и (11), убеждаемся, что обе системы совместны, то есть, никакое $k$ не удовлетворяет определению 4, следовательно, у функции $f$ нет правого барьера. Аналогично, воспользовавшись парой (13), убеждаемся в отсутствии у $f$ левого барьера. Следовательно, $f \in \mathscr{P}_{5}$ является функцией без барьера. 
С учетом полученных выше результатов, перейдем к более детальному изучению функций из $\Phi_{n}$ с правым барьером длины $k, 2 \leqslant k \leqslant n$.

Применим обозначения (9) к функции $f$, преобразуем уравнения систем (10), (11), после чего легко убедиться в справедливости следующего утверждения.

Теорема 8. Функция $f \in \mathscr{F}_{n}$ является функиией с правым барьером длины $k, 2 \leqslant k \leqslant n$, тогда и только тогда, когда система уравнений

$$
\begin{gathered}
f_{1}\left(x_{1}, \ldots, x_{n-1}\right)=0, \\
f_{0}\left(x_{2}, \ldots, x_{n-1}, 0\right) \\
\oplus f_{0}\left(x_{2}, \ldots, x_{n-1}, 1\right) \\
\oplus y_{n+1} f_{1}\left(x_{2}, \ldots, x_{n-1}, 0\right) \\
\oplus z_{n+1} f_{1}\left(x_{2}, \ldots, x_{n-1}, 0\right)=0, \\
\cdots \\
f_{0}\left(x_{k-1}, \ldots, x_{n-1}, 0, y_{n+1}, \ldots, y_{n+k-3}\right) \\
\oplus f_{0}\left(x_{k-1}, \ldots, x_{n-1}, 1, z_{n+1}, \ldots, z_{n+k-3}\right) \\
\oplus y_{n+k-2} f_{1}\left(x_{k-1}, \ldots, x_{n-1}, 0, y_{n+1}, \ldots, y_{n+k-3}\right) \\
\oplus z_{n+k-2} f_{1}\left(x_{k-1}, \ldots, x_{n-1}, 1, z_{n+1}, \ldots, z_{n+k-3}\right)=0
\end{gathered}
$$

имеет решение, причем для любого решения системы (14) выполнень условия

$$
\begin{aligned}
f_{1}\left(x_{k}, \ldots, x_{n-1}, 0, y_{n+1}, \ldots, y_{n+k-2}\right) & =0, \\
f_{1}\left(x_{k}, \ldots, x_{n-1}, 1, z_{n+1}, \ldots, z_{n+k-2}\right) & =0, \\
f_{0}\left(x_{k}, \ldots, x_{n-1}, 0, y_{n+1}, \ldots, y_{n+k-2}\right) \oplus f_{0}\left(x_{k}, \ldots, x_{n-1}, 1, z_{n+1}, \ldots, z_{n+k-2}\right) & =1 .
\end{aligned}
$$

В качестве первого простого примера применения теоремы 8 приведем критерий того, что функция $f \in \mathscr{F}_{n}$ имеет правый барьер длины $k=2$ : если $f \notin \mathscr{R}_{n}$ и для всех $\left(x_{1}, \ldots, x_{n-1}\right) \in \mathrm{V}_{n-1}$ справедливо равенство $f_{1}\left(x_{1}, \ldots, x_{n-1}\right)=0$, то

$$
\begin{aligned}
f_{1}\left(x_{2}, \ldots, x_{n-1}, 0\right) & =0, \\
f_{1}\left(x_{2}, \ldots, x_{n-1}, 1\right) & =0, \\
f_{01}\left(x_{2}, \ldots, x_{n-1}\right) & =1 .
\end{aligned}
$$

Очевидно, что этот критерий допускает многократную итерацию, после которой приходим к следующему утверждению.

Лемма 3. Булева функция $f \in \mathscr{F}_{n}$ имеет правый барьер длины $k=2$ тогда и только тогда, когда $f_{1}\left(x_{1}, \ldots, x_{n-1}\right) \equiv 0 u f_{01}\left(x_{2}, \ldots, x_{n-1}\right) \equiv 1$.

Рассмотренный пример приводит к мысли попытаться найти другие случаи, когда из условий (14), (15) можно выделить условия, допускающие многократную итерацию. Для этого применим следующий прием: разобьем все решения системы (14) на определенные классы так, чтобы для всех решений из одного класса условие (15) приводило к ситуации, допускающей итерацию.

Вначале отметим полезные для дальнейшего особенности системы (14). Относительно неизвестных $y_{n+1}, \ldots, y_{n+k-2}, z_{n+1}, \ldots, z_{n+k-2}$ система (14) является треугольной каждое следующее уравнение системы добавляет в нее одно неизвестное $y$ и одно неизвестное $z$. Более того, первое вхождение неизвестных $y_{n+j}, z_{n+j}, 1 \leqslant j \leqslant k-2$, в систему (14) является линейным с коэффициентами $f_{1}\left(x_{j+1}, \ldots, x_{n-1}, 0, y_{n+1}, \ldots, y_{n+j-1}\right)$, 
$f_{1}\left(x_{j+1}, \ldots, x_{n-1}, 1, z_{n+1}, \ldots, z_{n+j-1}\right)$ соответственно. Тем самым при различных условиях на коэффициенты можно выражать неизвестные $y_{n+j}, z_{n+j}$ через неизвестные $x_{2}, \ldots, x_{n-1}, y_{n+1}, \ldots, y_{n+j-1}, z_{n+1}, \ldots, z_{n+j-1}$ или путем последовательного (рекуррентного) выражения - только через переменные $x_{2}, \ldots, x_{n-1}$. Правда, при этом, как легко видеть, будут и ситуации, когда некоторые неизвестные $y_{n+j}, z_{n+j}$ произвольны.

Рассмотрим более подробно, к какому эквивалентному виду можно преобразовать $j$-е уравнение системы (14), $1 \leqslant j \leqslant k-2$ :

$$
\begin{aligned}
& f_{0}\left(x_{j+1}, \ldots, x_{n-1}, 0, y_{n+1}, \ldots, y_{n+j-1}\right) \\
& \quad \oplus f_{0}\left(x_{j+1}, \ldots, x_{n-1}, 1, z_{n+1}, \ldots, z_{n+j-1}\right) \\
& \quad \oplus y_{n+j} f_{1}\left(x_{j+1}, \ldots, x_{n-1}, 0, y_{n+1}, \ldots, y_{n+j-1}\right) \\
& \quad \oplus z_{n+j} f_{1}\left(x_{j+1}, \ldots, x_{n-1}, 1, z_{n+1}, \ldots, z_{n+j-1}\right)=0 .
\end{aligned}
$$

Разобьем все возможные ситуации для зависимости неизвестных $y_{n+j}, z_{n+j}$ от предыдущих неизвестных $x_{j+1}, \ldots, x_{n-1}, y_{n+1}, \ldots, y_{n+j-1}, z_{n+1}, \ldots, z_{n+j-1}$ на три случая.

Случай о. Здесь

$$
f_{1}\left(x_{j+1}, \ldots, x_{n-1}, 0, y_{n+1}, \ldots, y_{n+j-1}\right)=f_{1}\left(x_{j+1}, \ldots, x_{n-1}, 1, z_{n+1}, \ldots, z_{n+j-1}\right)=0 .
$$

В этом случае уравнение (16) эквивалентно системе

$$
\begin{aligned}
& y_{n+j}, z_{n+j} \text { произвольны, } \\
& f_{0}\left(x_{j+1}, \ldots, x_{n-1}, 0, y_{n+1}, \ldots, y_{n+j-1}\right) \oplus f_{0}\left(x_{j+1}, \ldots, x_{n-1}, 1, z_{n+1}, \ldots, z_{n+j-1}\right) \\
& \quad \equiv F_{j}\left(x_{2}, \ldots, x_{n-1}, y_{n+1}, \ldots, y_{n+j-1}, z_{n+1}, \ldots, z_{n+j-1}\right)=0 .
\end{aligned}
$$

Случай 1. Здесь

$$
f_{1}\left(x_{j+1}, \ldots, x_{n-1}, 0, y_{n+1}, \ldots, y_{n+j-1}\right) \oplus f_{1}\left(x_{j+1}, \ldots, x_{n-1}, 1, z_{n+1}, \ldots, z_{n+j-1}\right)=1 .
$$

В этом случае уравнение (16) эквивалентно одной из систем (в зависимости от того, какой из коэффициентов равен единице):

$$
\begin{aligned}
& y_{n+j} \text { произвольно, } \\
& z_{n+j}=F_{j}\left(x_{2}, \ldots, x_{n-1}, y_{n+1}, \ldots, y_{n+j-1}, z_{n+1}, \ldots, z_{n+j-1}\right)
\end{aligned}
$$

или

$$
\begin{aligned}
& z_{n+j} \text { произвольно, } \\
& y_{n+j}=F_{j}\left(x_{2}, \ldots, x_{n-1}, y_{n+1}, \ldots, y_{n+j-1}, z_{n+1}, \ldots, z_{n+j-1}\right) .
\end{aligned}
$$

Случай 2. Здесь

$$
f_{1}\left(x_{j+1}, \ldots, x_{n-1}, 0, y_{n+1}, \ldots, y_{n+j-1}\right)=f_{1}\left(x_{j+1}, \ldots, x_{n-1}, 1, z_{n+1}, \ldots, z_{n+j-1}\right)=1 \text {. }
$$

В этом случае уравнение (16) эквивалентно уравнению

$$
y_{n+j} \oplus z_{n+j}=F_{j}\left(x_{2}, \ldots, x_{n-1}, y_{n+1}, \ldots, y_{n+j-1}, z_{n+1}, \ldots, z_{n+j-1}\right) .
$$


Если теперь с этих позиций посмотреть на систему (14), то в зависимости от того, какой из выделенных случаев реализуется для разных уравнений системы, возможно $2^{2(k-2)}$ вариантов, на которые можно разбить решения системы (14). Для каждого из вариантов систему можно заменить на более удобную эквивалентную. Теперь заметим, что в соответствии с условием (15) для коэффициентов $k$-го уравнения будет выполнен случай 0 , правда, в условие (15) входит еще и уравнение

$$
F_{k-1}\left(x_{2}, \ldots, x_{n-1}, y_{n+1}, \ldots, y_{n+k-2}, z_{n+1}, \ldots, z_{n+k-2}\right)=1 .
$$

Если с учетом этих замечаний найти такие ситуации, когда из условий (14), (15) удастся выделить итерационную процедуру, то это позволит сделать некоторые выводы о строении функций с правым барьером длины $k$.

Рассмотрим вначале более подробно ситуацию, когда для каждого уравнения системы (14) (кроме, естественно, первого) реализуется случай 0 или 1. Пользуясь описанными выше приемами, преобразуем систему (14) к эквивалентному, но более удобному для анализа виду. Каждое уравнение системы (14) будем последовательно заменять системой, которую в дальнейшем будем называть блоком преобразованной системы (14). Записывать блоки будем для наглядности в два столбца: слева - условия на $f_{1}$, справа - условия на $y_{j}, z_{j}$ и $f_{0}$. Блок, соответствующий $j$-му уравнению из случая 1 , будет выглядеть так:

$$
\begin{array}{cl}
f_{1}\left(x_{j+1}, \ldots, x_{n-1}, 0, y_{n+1}, \ldots, y_{n+j-1}\right)=0, & y_{n+j} \text { произвольно, } \\
y_{n+i_{1}}, \ldots, y_{n+i_{l}} \text { произвольны, } & z_{n+j}=F_{n+j} . \\
f_{1}\left(x_{j+1}, \ldots, x_{n-1}, 1, z_{n+1}, \ldots, z_{n+j-1}\right)=1, & \\
z_{n+m_{1}}, \ldots, z_{n+m_{s}} \text { произвольны, } &
\end{array}
$$

или так:

$$
\begin{array}{cl}
f_{1}\left(x_{j+1}, \ldots, x_{n-1}, 0, y_{n+1}, \ldots, y_{n+j-1}\right)=1, & z_{n+j} \text { произвольно, } \\
y_{n+i_{1}}, \ldots, y_{n+i_{l}} \text { произвольны, } & y_{n+j}=F_{n+j} . \\
f_{1}\left(x_{j+1}, \ldots, x_{n-1}, 1, z_{n+1}, \ldots, z_{n+j-1}\right)=0, & \\
z_{n+m_{1}}, \ldots, z_{n+m_{s}} \text { произвольны, } &
\end{array}
$$

Правую часть блока, соответствующего $j$-му уравнению из случая 0 , дополнительно преобразуем следующим образом. В уравнении

$$
f_{0}\left(x_{j+1}, \ldots, x_{n-1}, 0, y_{n+1}, \ldots, y_{n+j-1}\right) \oplus f_{0}\left(x_{j+1}, \ldots, x_{n-1}, 1, z_{n+1}, \ldots, z_{n+j-1}\right)=0
$$

предыдущие неизвестные $y_{n+i}, z_{n+i}$ заменим на $F_{n+i}$, если так было в $i$-м блоке, или на 0 и одно из двух возможных дополнительных уравнений

$$
\begin{aligned}
& D^{r(i, j)} f_{0}\left(x_{j+1}, \ldots, x_{n-1}, 0, y_{n+1}, \ldots, y_{n+j-1}\right)=0, \quad y_{n+i} \text { произвольно, } \\
& D^{r(i, j)} f_{0}\left(x_{j+1}, \ldots, x_{n-1}, 1, z_{n+1}, \ldots, z_{n+j-1}\right)=0, \quad z_{n+i} \text { произвольно }
\end{aligned}
$$

(здесь $r(i, j)$ - номер аргумента функции $f_{0}\left(u_{1}, \ldots, u_{n-1}\right)$, который замещается неизвестным $y_{n+i}$ или $\left.z_{n+i}\right)$.

Поскольку по условию для всех $i=1, \ldots, j-1$ предыдущие уравнения либо из случая 0 , либо из случая 1 , для каждого $i=1, \ldots, j-1$ по крайней мере одна из указанных производных равна нулю. Кроме того, все неизвестные $y_{n+1}, \ldots, y_{n+j-1}$, $z_{n+1}, \ldots, z_{n+j-1}$ в уравнении (23) заменены либо на $F$, либо на 0. 
Заменим описанным способом все уравнения системы (14) на блоки. Применим условие (15) и добавим к системе блоков (14) аналогичную систему блоков для уравнений из (15) и условие: $y_{n+k-1}, z_{n+k-1}$ любые. Полученная объединенная система блоков (14), (15) по условию имеет решение. Выберем из нее подсистему, похожую на (14), которая, естественно, также будет иметь решение. Выбросим первый блок - уравнение $f_{1}\left(x_{1}, \ldots, x_{n-1}\right)=0$. Первым блоком новой системы будем считать уравнение $f_{1}\left(x_{2}, \ldots, x_{n-1}, 0\right)=0$ или $f_{1}\left(x_{2}, \ldots, x_{n-1}, 1\right)=0$, одно из них заведомо есть во втором блоке по условию. Если выбрано уравнение $f_{1}\left(x_{2}, \ldots, x_{n-1}, \varepsilon\right)=0, \varepsilon \in \mathbf{F}_{2}$, то из системы блоков выбрасываются все уравнения, в аргументах которых после $x_{n-1}$ следует $\varepsilon \oplus 1$. Ясно, что после всех указанных преобразований мы получим новую совместную систему блоков для (14), которая обладает следующими свойствами:

- все уравнения (блоки) системы принадлежат случаям 0 или 1 , причем случаев 0 на один больше, чем было раньше, за счет нового последнего уравнения (блока);

- система начинается с неизвестной $x_{2}$ (а не $x_{1}$ ), а заканчивается уже произвольными значениями неизвестных $y_{n+k-1}, z_{n+k-1}$.

К полученной системе применяем новое условие $(14) \rightarrow(15)$, применяем такую же процедуру выбора новой совместной подсистемы и так далее. Теперь практически очевидно, что после какого-то числа итераций мы придем к выводу, что $f_{1} \equiv 0$, а это противоречит предположению $f \in \Phi_{n}$.

Тем самым доказано следующее утверждение.

Теорема 9. Пусть $f \in \Phi_{n}$ имеет правыцй барьер длины $k, 3 \leqslant k \leqslant n$. Тогда для любого решения системы (14) по крайней мере для одного $j, 1 \leqslant j \leqslant k-2$, выполнено условие

$$
f_{1}\left(x_{j+1}, \ldots, x_{n-1}, 0, y_{n+1}, \ldots, y_{n+j-1}\right)=f_{1}\left(x_{j+1}, \ldots, x_{n-1}, 1, z_{n+1}, \ldots, z_{n+j-1}\right)=1 .
$$

Вместе с тем, как легко видеть, и в ситуации, указанной в теореме 9, можно таким же образом, как описано выше, итерировать условия наличия барьера (уравнения (14), (15)) до тех пор, пока это возможно. Итерация невозможна, когда уже второе уравнение (блок) относится к случаю 2. Тем самым, в дальнейшем надо в первую очередь проанализировать ситуацию, когда если система (14) имеет решение, то для любого решения выполнено условие

$$
f_{1}\left(x_{2}, \ldots, x_{n-1}, 0\right)=f_{1}\left(x_{2}, \ldots, x_{n-1}, 1\right)=1 .
$$

Рассмотрим теперь более подробно случай правого барьера длины 3. Из предыдущего вытекает, что функция $f \in \mathscr{F}_{n}$ имеет правый барьер длины 3 тогда и только тогда, когда выполнены следующие условия.

(1) Если система уравнений

$$
\begin{aligned}
f_{1}\left(x_{1}, \ldots, x_{n-1}\right) & =0, \\
f_{01}\left(x_{2}, \ldots, x_{n-1}\right) \oplus y_{n+1} f_{1}\left(x_{2}, \ldots, x_{n-1}, 0\right) \oplus z_{n+1} f_{1}\left(x_{2}, \ldots, x_{n-1}, 1\right) & =0
\end{aligned}
$$

имеет решение, то

$$
\begin{aligned}
& f_{1}\left(x_{2}, \ldots, x_{n-1}, 0\right)=1 \\
& f_{1}\left(x_{2}, \ldots, x_{n-1}, 1\right)=1 .
\end{aligned}
$$


(2) Для любого решения системы (25) выполнены условия

$$
\begin{aligned}
f_{1}\left(x_{3}, \ldots, x_{n-1}, 0, y_{n+1}\right) & =0, \\
& f_{1}\left(x_{3}, \ldots, x_{n-1}, 1, z_{n+1}\right)=0, \\
f_{0}\left(x_{3}, \ldots, x_{n-1}, 0, y_{n+1}\right) \oplus f_{0}\left(x_{3}, \ldots, x_{n-1}, 1, z_{n+1}\right) & =1 .
\end{aligned}
$$

Легко видеть, что условие 1 эквивалентно следующему условию.

(3) Если $f_{1}\left(x_{1}, \ldots, x_{n-1}\right)=0$, то $f_{11}\left(x_{2}, \ldots, x_{n-1}\right)=0$, и при этом, если $f_{10}\left(x_{2}, \ldots, x_{n-1}\right)=0$, то $f_{01}\left(x_{2}, \ldots, x_{n-1}\right)=1$.

Теперь условие 2 можно преобразовать к следующему эквивалентному условию.

(4) Если $f_{1}\left(x_{1}, \ldots, x_{n-1}\right)=0$, то $f_{11}\left(x_{2}, \ldots, x_{n-1}\right)=0$, и при этом, если $f_{10}\left(x_{2}, \ldots, x_{n-1}\right)=1$, то для

$$
y_{n+1} \oplus z_{n+1}=f_{01}\left(x_{2}, \ldots, x_{n-1}\right)
$$

выполнены условия (26).

Поскольку и $y_{n+1}$, и $z_{n+1}$ смогут принимать значения 0,1 , но связаны условием (27), условие (26) можно заменить следующим эквивалентным условием:

$$
\begin{aligned}
& f_{1}\left(x_{3}, \ldots, x_{n-1}, 0,0\right)=0, \\
& f_{1}\left(x_{3}, \ldots, x_{n-1}, 0,1\right)=0 \text {, } \\
& f_{1}\left(x_{3}, \ldots, x_{n-1}, 1,0\right)=0 \text {, } \\
& f_{1}\left(x_{3}, \ldots, x_{n-1}, 1,1\right)=0, \\
& f_{01}\left(x_{3}, \ldots, x_{n-1}, 0\right) \oplus f_{01}\left(x_{3}, \ldots, x_{n-1}, 1\right)=0, \\
& f_{00}\left(x_{3}, \ldots, x_{n-1}, 0\right) \oplus f_{00}\left(x_{3}, \ldots, x_{n-1}, 1\right) \oplus f_{01}\left(x_{2}, \ldots, x_{n-1}\right) f_{01}\left(x_{3}, \ldots, x_{n-1}, 0\right)=1 \text {. }
\end{aligned}
$$

Анализируя теперь цепочки условий, содержащиеся в условиях 3 , 4, замечаем, что из них можно выделить условия только на функцию $f_{1}$, а после выполнения этих условий добиваться выполнения условий на функцию $f_{0}$. Вначале выделяем цепочку условий, которая допускает итерацию: если $f_{1}\left(x_{1}, \ldots, x_{n-1}\right)=0$, то $f_{11}\left(x_{2}, \ldots, x_{n-1}\right)=0$ и затем $f_{11}\left(x_{3}, \ldots, x_{n-1}, 0\right)=f_{11}\left(x_{3}, \ldots, x_{n-1}, 1\right)=0$.

Отсюда делаем вывод, что

$$
f_{11}\left(x_{2}, \ldots, x_{n-1}\right) \equiv 0
$$

и поэтому

$$
f_{1}\left(x_{1}, \ldots, x_{n-1}\right) \equiv f_{10}\left(x_{1}, \ldots, x_{n-2}\right) .
$$

Теперь с учетом полученных тождеств выделяем следующую цепочку условий: если $f_{10}\left(x_{1}, \ldots, x_{n-2}\right)=0$ и $f_{10}\left(x_{2}, \ldots, x_{n-1}\right)=1$, то

$$
\begin{aligned}
& f_{10}\left(x_{3}, \ldots, x_{n-1}, 0\right)=0, \\
& f_{10}\left(x_{3}, \ldots, x_{n-1}, 1\right)=0 .
\end{aligned}
$$

Поскольку $f_{10}\left(x_{1}, \ldots, x_{n-2}\right) \not \equiv 1$, рассуждая от противного и итерируя условие в обратном направлении, приходим к выводу, что если $f_{10}\left(x_{1}, \ldots, x_{n-2}\right)=1$, то $f_{10}\left(x_{2}, \ldots, x_{n-2}, 0\right)=0, f_{10}\left(x_{2}, \ldots, x_{n-2}, 1\right)=0$.

Суммируя проведенные рассуждения, приходим к следующему критерию. 
Таблица 1.

\begin{tabular}{lccc} 
& $f_{1}$ & $\left(x_{1}, x_{2}, x_{3}\right): f=1$ & Число подходящих $f_{0}$ \\
\hline 1 & $x_{1} x_{2}\left(x_{3} \oplus 1\right)$ & $(1,1,0)$ & 16 \\
2 & $x_{1}\left(x_{2} \oplus 1\right) x_{3}$ & $(1,0,1)$ & 15 \\
3 & $\left(x_{1} \oplus 1\right) x_{2} x_{3}$ & $(0,1,1)$ & 15 \\
4 & $\left(x_{1} \oplus 1\right) x_{2}\left(x_{3} \oplus 1\right)$ & $(0,1,0)$ & 1 \\
5 & $x_{1}\left(x_{2} \oplus 1\right)\left(x_{3} \oplus 1\right)$ & $(1,0,0)$ & 1 \\
6 & $\left(x_{1} \oplus 1\right)\left(x_{2} \oplus 1\right) x_{3}$ & $(0,0,1)$ & 0 \\
7 & $x_{1}\left(x_{2} \oplus 1\right)$ & $(1,0,0),(1,0,1)$ & 10 \\
8 & $\left(x_{2} \oplus 1\right) x_{3}$ & $(0,0,1),(1,0,1)$ & 20 \\
9 & $x_{2}\left(x_{3} \oplus 1\right)$ & $(0,1,0),(1,1,0)$ & 28 \\
10 & $\left(x_{1} \oplus 1\right) x_{2}$ & $(0,1,0),(0,1,1)$ & 6 \\
11 & $\left(x_{1} \oplus x_{2} \oplus 1\right)\left(x_{2} \oplus x_{3}\right)$ & $(1,1,0),(0,0,1)$ & 10 \\
12 & $\left(x_{1} \oplus x_{2}\right)\left(x_{2} \oplus x_{3} \oplus 1\right)$ & $(0,1,1),(1,0,0)$ & 10 \\
\hline Всего & & &
\end{tabular}

Таблица 2.

\begin{tabular}{|r|r|r|r|r|r|r|}
\hline$n$ & 1 & \multicolumn{1}{|c|}{2} & \multicolumn{1}{|c|}{3} & \multicolumn{1}{|c|}{4} & 5 & $+\infty$ \\
\hline 3 & 28 & 2 & 0 & 0 & 0 & 0 \\
\hline 4 & 496 & 22 & 64 & 0 & 0 & 0 \\
\hline 5 & 130816 & 476 & 8146 & 3136 & 228 & 216 \\
\hline
\end{tabular}

Теорема 10. Функиия $f \in \Phi_{n} \backslash \mathscr{R}_{n}$ имеет правый барьер длинь 3 тогда и только тогда, когда выполнены следующие условия:

(1) $f_{11}\left(x_{2}, \ldots, x_{n-1}\right) \equiv 0$;

(2) если $f_{10}\left(x_{1}, \ldots, x_{n-2}\right)=1$, то

$$
\begin{aligned}
f_{10}\left(x_{2}, \ldots, x_{n-2}, 0\right) & =f_{10}\left(x_{2}, \ldots, x_{n-2}, 1\right)=0 \\
f_{10}\left(0, x_{1} \ldots, x_{n-3}\right) & =f_{10}\left(1, x_{1}, \ldots, x_{n-3}\right)=0
\end{aligned}
$$

(3) если $f_{10}\left(x_{1}, \ldots, x_{n-2}\right)=f_{10}\left(x_{2}, \ldots, x_{n-1}\right)=0$, то

$$
f_{01}\left(x_{2}, \ldots, x_{n-1}\right)=1
$$

(4) если $f_{10}\left(x_{1}, \ldots, x_{n-2}\right)=1$, то

$$
\begin{aligned}
f_{011}\left(x_{2}, \ldots, x_{n-2}\right) & =0, \\
f_{001}\left(x_{2}, \ldots, x_{n-2}\right) \oplus f_{01}\left(x_{1}, \ldots, x_{n-2}\right) f_{01}\left(x_{2}, \ldots, x_{n-2}, 0\right) & =1 .
\end{aligned}
$$

Множество функций $f_{1}$, для которых можно пытаться искать функции $f_{0}$, чтобы построить функцию с правым барьером длины 3, описывается условиями 1, 2 теоремы 10. Для случая $n=5$ все эти функции легко выписать вручную, просто перебирая возможное размещение единиц на булевом кубе размерности 3. Результаты приведены в табл. 1.

Алгоритмом полного перебора функций, построения их графов сдвигов, изучения длин путей и наличия циклов получены числа совершенно уравновешенных функций из $\mathscr{F}_{n}, n=3,4,5$, с барьером и без барьера, приведенные в табл. 2. 
Теорема 11. Пусть $f \in \mathscr{F}_{n}-$ функция с правым (левым) барьером длины $k, k<n, a$ $g \in \mathscr{F}_{n-k}-$ произвольная функция. Тогда функция

$$
h\left(x_{1}, \ldots, x_{n}\right)=f\left(x_{1}, \ldots, x_{n}\right) \oplus g\left(x_{1}, \ldots, x_{n-k}\right)
$$

(соответственно, $\left.h\left(x_{1}, \ldots, x_{n}\right)=f\left(x_{1}, \ldots, x_{n}\right) \oplus g\left(x_{k+1} \ldots, x_{n}\right)\right)$ тоже является функияией с правым (левым) барьером длины $k$.

Доказательство. Проведем доказательство для правого барьера (для левого барьера доказательство совершенно аналогично). Подставляя функцию $h$ в системы (10), (11), получим системы

$$
\begin{aligned}
f\left(x_{1}, \ldots, x_{n-1}, 0\right) & \oplus g\left(x_{1}, \ldots, x_{n-k}\right) \\
& =f\left(x_{1}, \ldots, x_{n-1}, 1\right) \oplus g\left(x_{1}, \ldots, x_{n-k}\right), \\
f\left(x_{2}, \ldots, x_{n-1}, 0, y_{n+1}\right) & \oplus g\left(x_{2}, \ldots, x_{n-k+1}\right) \\
& =f\left(x_{2}, \ldots, x_{n-1}, 1, z_{n+1}\right) \oplus g\left(x_{2}, \ldots, x_{n-k+1}\right), \\
\ldots & \\
f\left(x_{k-1}, \ldots, x_{n-1}, 0, y_{n+1}, \ldots, y_{k+n-2}\right) & \oplus g\left(x_{k-1}, \ldots, x_{n-2}\right) \\
= & f\left(x_{k-1}, \ldots, x_{n-1}, 1, z_{n+1}, \ldots, z_{k+n-2}\right) \\
& \oplus g\left(x_{k-1}, \ldots, x_{n-2}\right)
\end{aligned}
$$

И

$$
\begin{aligned}
f\left(x_{1}, \ldots, x_{n-1}, 0\right) & \oplus g\left(x_{1}, \ldots, x_{n-k}\right) \\
& =f\left(x_{1}, \ldots, x_{n-1}, 1\right) \oplus g\left(x_{1}, \ldots, x_{n-k}\right), \\
f\left(x_{2}, \ldots, x_{n-1}, 0, y_{n+1}\right) & \oplus g\left(x_{2}, \ldots, x_{n-k+1}\right) \\
& =f\left(x_{2}, \ldots, x_{n-1}, 1, z_{n+1}\right) \oplus g\left(x_{2}, \ldots, x_{n-k+1}\right), \\
& \ldots \\
f\left(x_{k}, \ldots, x_{n-1}, 0, y_{n+1}, \ldots, y_{k+n-1}\right) & \oplus g\left(x_{k}, \ldots, x_{n-1}\right) \\
& =f\left(x_{k}, \ldots, x_{n-1}, 1, z_{n+1}, \ldots, z_{k+n-1}\right) \\
& \oplus g\left(x_{k}, \ldots, x_{n-1}\right) .
\end{aligned}
$$

В каждом из уравнений систем (29)-(30) значение функции $g$ берется в правой и левой частях от одних и тех же переменных, то есть эти две системы, выписанные для функции $h$, эквивалентны аналогичным двум системам для функции $f$. Следовательно, $h$ имеет правый барьер той же длины, что и $f$.

Теорема 11 в совокупности с замечанием 1 дает возможность провести классификацию $\mathscr{P}_{n}$ относительно преобразований $\gamma_{0}-\gamma_{2}$ и указанного в теореме 11 преобразования. С помощью теоремы 7 несложно получить алгоритм проверки булевой функции на совершенную уравновешенность, имеющий сложность $O\left(4^{n}\right)$ операций, с помощью его практической реализации была получена классификация $\mathscr{P}_{\mathscr{P}_{4}}$ и $\mathscr{P} \mathscr{B}_{5}$ относительно упомянутых преобразований. Полученная классификация $\Phi_{4} \cap\left(\mathscr{P}_{\mathscr{B}_{4}} \backslash\left(\mathscr{L}_{4} \cup \mathscr{R}_{4}\right)\right)$, $\Phi_{5} \cap\left(\mathscr{P}_{5} \backslash\left(\mathscr{L}_{5} \cup \mathscr{R}_{5}\right)\right)$ находится в Приложении. 


\section{5. Заключение}

В настоящей работе предложен новый подход к исследованию свойств совершенно уравновешенных булевых функций. В рамках этого подхода развиваются методы исследований, основанные на новом параметре, названном барьером функции. Этот параметр связан с особенностями функционирования соответствующего кодирующего устройства. Использование барьера позволяет естественным образом разбивать функции из $\mathscr{P} \mathscr{B}_{n}$ на классы с целью их дальнейшего изучения. Приведенные в статье результаты показывают перспективность исследований как по классификации таких функций, так и по описанию новых широких классов функций из $\mathscr{P}_{\mathscr{B}_{n}}$. Кроме того, выявленное у функций из $\mathscr{P} \mathscr{\Re}_{n}$ новое специфическое свойство показывает необходимость глубоких теоретических исследований в области криптографических приложений совершенно уравновешенных булевых функций.

В заключение приведем основные, перспективные на взгляд авторов, направления исследований совершенно уравновешенных булевых функций:

- изучение алгебраических и комбинаторных свойств функций с барьером;

- развитие аналитического и алгоритмического математического аппарата, необходимого для проведения классификации совершенно уравновешенных булевых функций от шести и более переменных;

- математическое обоснование перспектив использования совершенно уравновешенных булевых функций с барьером или без барьера в криптографических приложениях.

\section{Список литературы}

1. Preparata F. P., Convolutional transformations of binary sequences: Boolean functions and their resynchronizing properties. IEEE Trans. Electron. Comput. (1966) 15, №6, 898-909.

2. Huffman D. A., Canonical forms for information-lossless finite-state logical machines. IRE Trans. Circuit Theory (1959) 5, 41-59.

3. Логачев О. А., Сальников А. А., Ященко В. В., Булевы функиии в теории кодирования и криптологии. МЦНМО, Москва, 2004.

4. Anderson R. J., Searching for the optimum correlation attack. Lecture Notes Computer Sci. (1995) 1008, 137-143.

5. Сумароков С. Н., Запреты двоичных функций и обратимость для одного класса кодирующих устройств. Обозрение прикладной и промышленной математики (1994) 1, №1, 33-55.

6. Golić D. J., On the security of nonlinear filter generators. Lecture Notes Computer Sci. (1996) 1039, 173-188.

7. Gouget A., Sibert H., Revisiting correlation-immunity in filter generators. Lecture Notes Computer Sci. (2007) 4876, 378-395.

8. Dichtl M., On nonlinear filter generators. Lecture Notes Computer Sci. (1997) 1267, 103-106.

9. Логачев О. А., Об одном классе совершенно уравновешенных булевых функций. В сб.: Материалы III Международной научной конференции по проблемам безопасности и противодействия терроризму. МЦНМО, Москва, 2008, с. 137-141.

10. Gill A., Introduction to the theory of finite-state machines. McGraw-Hill, New York, 1962.

11. Шеннон К., Математическая теория связи. В сб.: Работы по теории информации и кибернетике. ИЛ, Москва, 1963, с. 243-332. 


\section{Приложение}

Представители классов эквивалентности множеств $\Phi_{4} \cap\left(\mathscr{P}_{4} \backslash\left(\mathscr{L}_{4} \cup \mathscr{R}_{4}\right)\right)$ и $\Phi_{5} \cap\left(\mathscr{P}_{5} \backslash\left(\mathscr{L}_{5} \cup \mathscr{R}_{5}\right)\right)$ относительно преобразований $\gamma_{0}-\gamma_{2}$ делятся на подмножества относительно длины барьера, которые затем классифицируются относительно преобразований, полученных в теореме 11. Для удобства при выборе представителей классов эквивалентности относительно преобразования $\gamma_{2}$ выбирались функции с длиной барьера, равной длине правого барьера.

$\Phi_{4} \cap\left(\mathscr{P}_{\mathscr{B}_{4}} \backslash\left(\mathscr{L}_{4} \cup \mathscr{R}_{4}\right)\right)$, барьер длины $k=3$ :

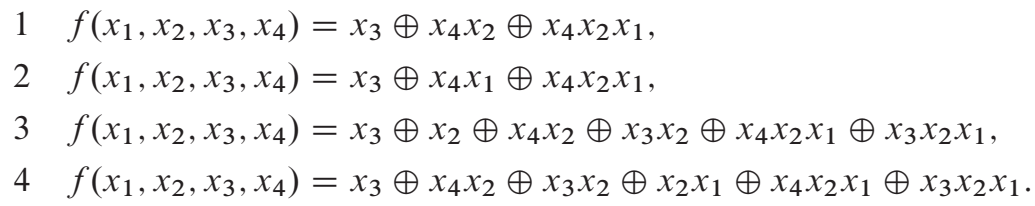

Далее все функции представлены вектором коэффициентов АНФ.

\section{$\Phi_{5} \cap\left(\mathscr{P}_{5} \backslash\left(\mathscr{L}_{5} \cup \mathscr{R}_{5}\right)\right)$, барьер длины $k=3:$}

$f_{1}: 00100000000000000000000001000100$

$f_{3}: 00100000000000000100000001000000$;

$f_{5}: 00100100000001000000000010000000$;

$f_{7}: 00100000010001000000000000001000$;

$f_{9}: 00100000010001000000000010000000$;

$f_{11}: 00100100000001000000000000001000$; $f_{13}: 00100000000000000100100001000000$; $f_{15}: 00100000000000000000110000000100$; $f_{17}: 00100000000011000000000000001100$; $f_{19}$ : 00101000000010000000010000000100 ; $f_{21}$ :00100100000001000000010001000000; $f_{23}: 00101000000001000000000000001100$; $f_{25}: 00100000000001000000100000001100$; $f_{27}: 00100000000010000000000001001100$; $f_{29}: 00100000010011000000000000001000$; $f_{31}$ : 00101000000001100000000000000110 ; $f_{33}: 00101000000000000000011000000110$; $f_{35}: 00101000010001000010000000100000$; $f_{37}: 00101000010001000010000000000010$; $f_{39}: 00100100000001000000010001001000$; $f_{41}: 00100000011001100000100000000000$; $f_{43}: 00100000010001000010100000000010$; $f_{2}: 001000000000000000000100100$;

$f_{4}: 00100000000001000000000000000100$; $f_{6}$ : 00100000000010000000000001000100 ; $f_{8}: 00100000000000000000000001001100$; $f_{10}: 00100000000000000100000001001000$; $f_{12}$ : 00100000000000000000010000001100 ; $f_{14}: 00100100000001000000100000000000$; $f_{16}: 00100000000000000000110000001100$; $f_{18}: 00100000000000000100100001001000$; $f_{20}: 00101000000001000000100000000100$; $f_{22}: 00100000000011000000100000000100$; $f_{24}: 00100000000001000100010001000000$; $f_{26}$ : 00101000000011000000000000000100 ; $f_{28}: 00101000000000000000100001000100$; $f_{30}: 00101000010001000000100000000000$; $f_{32}$ : 00101000011000000000000001100000 ; $f_{34}: 00101000000000000000000001100110$; $f_{36}: 00101000000000000000100001001100$; $f_{38}: 00101000000010000000100001000100$; $f_{40}: 00100000010001000010100000100000$; $f_{42}: 00100000000000000000100001100110$; $f_{44}: 00100000010010100000000001000010$; 
$f_{45}: 00100000010011000000000001000100$; $f_{47}: 00100000011000000000100001100000$; $f_{49}: 00101000000010000000110000000100$; $f_{51}: 00101100000011000000000000001000$; $f_{53}: 00100000000010000100001001000010$; $f_{55}: 00100000000011100000000000000110$; $f_{57}: 00100000000001100000000000001110$; $f_{59}: 00101100001001000000000000101000$; $f_{61}: 00101000010011000010000000000010$; $f_{63}: 00100000011001100000100000001000$; $f_{65}: 00101100001001000000100000100000$; $f_{67}: 00100000000010000000011000001110$; $f_{69}: 00100000000010000100001001001010$; $f_{71}: 00100000000010000000111000000110$; $f_{73}: 00101110000001100000000000001000$; $f_{75}: 00101000000000000110000001101000$; $f_{77}: 00100000000001000100110001001000$; $f_{79}: 00101000010001000010000000001010$; $f_{81}: 00101000000000000000000001101110$; $f_{83}: 00101000010001000010000000101000$; $f_{85}: 00100110000011100000000010000000$; $f_{87}: 00100000000011100000100000001110$; $f_{89}: 00100000000010000000100001101110$; $f_{91}: 00101000010000100000100001001010$; $f_{93}: 00101000000001100000100000001110$; $f_{95}: 00101000000000000000111000001110$; $f_{97}: 00101000010011000010000000101000$; $f_{99}: 00100000010011000010100000001010$; $f_{101}$ : 00100100000001000000110001100010 ; $f_{103}: 00100000011001100010100000100000$; $f_{105}$ :00101000000011100000000000001110; $f_{107}$ :00100100001011000000001000100010; $f_{109}$ :00100000000011100100010001000010; $f_{111}$ :00101000010010100000100001000010; $f_{113}$ : 00100000011001100010100000101000 ; $f_{115}$ : 00101100000011000000110001001000 ; $f_{117}$ : 00101100000011000000010001100010 ; $f_{119}$ :00101100001001000000001000101010; $f_{121}$ :00100100001011000000101000100010; $f_{46}: 00100000000000000100110001000100$; $f_{48}: 00100000000001100000100000000110$; $f_{50}: 00101000000010000000010000001100$; $f_{52}: 00101100001001000000000000100000$; $f_{54}: 00100000000010000000011000000110$; $f_{56}: 00100000010000100000000001001010$; $f_{58}: 00100000000011000100010001001000$; $f_{60}: 00100000010001000010100000001010$ $f_{62}: 00101000000010000000100001001100$; $f_{64}: 00100000000000000000100001101110$; $f_{66}: 00101000000010000000000001100110$; $f_{68}: 00101000000000000000111000000110$; $f_{70}: 00100100001011000000000000101000$; $f_{72}: 00100110000011100000100000000000$; $f_{74}: 00101000000000000000011000001110$ $f_{76}: 00100000000010000100101001000010$; $f_{78}: 00100000010011000010100000000010$ $f_{80}: 00100000000010000000100001100110$ $f_{82}: 00101000011001100000000000001000$; $f_{84}: 00101000011001100000000010000000$ $f_{86}: 00100000000010000100101001001010$; $f_{88}: 00100000000010000000111000001110$ $f_{90}: 00101000011001100010000000000010$ $f_{92}: 00101000011001100010000000100000$; $f_{94}$ : 00101110000001100000100000001000 ; $f_{96}: 00101000000000000110100001101000$; $f_{98}: 00100000011001100010100000000010$; $f_{100}: 00101000000010000000000001101110$; $f_{102}: 00101000010011000010000000001010$; $f_{104}: 00100000000001100100110001000010$; $f_{106}: 00101100001001000000001000100010$; $f_{108}: 00100110001011100000000000100000$; $f_{110}: 00100000000001100100010001001010$; $f_{112}: 00101000000011100000100000000110$; $f_{114}: 00101100001001000000101000100010$ $f_{116}: 00101000011011100010000000000010$ $f_{118}: 00100110001011100000100000100000$ $f_{120}: 00100100000001000000110001101010$; $f_{122}: 00101110001001100000000000101000$; 
$f_{123}: 00101000011001100010000000101000$; $f_{125}$ :00101110000001100000011001100000; $f_{127}$ :00101100000011000000010001101010; $f_{129}: 00100110000011100000111001100000$; $f_{131}: 00101000000001100110111001101000$; $f_{124}: 00101000000001100110011001100000$ $f_{126}: 00100000000011100100110001001010$ $f_{128}: 00101000011011100010000000101000$ $f_{130}: 00101110000001100000011001101000$ $f_{132}: 00101000000011100110111001100000$.

\section{$\Phi_{5} \cap\left(\mathscr{P} \mathscr{B}_{5} \backslash\left(\mathscr{L}_{5} \cup \mathscr{R}_{5}\right)\right)$, барьер длины $k=4$ :}

$f_{1}: 00100000000000000100000000000100$; $f_{3}: 00100000000000000100000010000100$; $f_{5}: 00100000100000000100100000000100$; $f_{7}: 00100000100000000100000000100110$; $f_{9}: 00100000000010000100001000000110$; $f_{11}: 00100000000000000100100000100110$; $f_{13}: 00100000000000000100100010100110$; $f_{15}: 00101000000010000110001000000100$; $f_{17}: 00101000100000000110000000100100$; $f_{19}$ : 00101000000000000110000000001110 ; $f_{21}: 00100000000010000100001010000110$; $f_{23}$ : 00100000000010000100001000001110 ; $f_{25}: 00100000100000000100000000101110$; $f_{27}: 00101000000000000110000010001110$; $f_{29}: 00100000100000000100000010101110$; $f_{31}: 00101000000000000110100000101100$; $f_{33}: 00101000000000000110100010100100$; $f_{35}: 00101000000010000110001010000100$; $f_{37}: 00101000100000000110100000000110$; $f_{39}: 00100000100010000100101000000110$; $f_{41}: 00100000000010000100001010001110$; $f_{43}: 00100000100010000100001010100100$; $f_{45}: 00100000011001100001100000011000$; $f_{47}: 00100000011001100001100010010000$; $f_{49}: 00101000000010000110001010001100$; $f_{51}: 00101000100000000110100000001110$; $f_{53}: 00100000010001000101010001010100$; $f_{55}: 00101100010110000000000001010100$; $f_{57}: 00100100110100000000000011010100$; $f_{59}$ : 00101000000000000110100010101100 ; $f_{61}: 00101000011001100001000010010000$; $f_{2}: 00100000000000000100000000001100$; $f_{4}: 00100000000000000100000010001100$; $f_{6}$ : 00101000000000000110000000000110 ; $f_{8}: 00100000100000000100100010000100$; $f_{10}$ : 00100000100000000100100000001100 ; $f_{12}: 00100000000000000100100000101110$; $f_{14}$ : 00100000100010000100001000100100 ; $f_{16}$ : 00100100010100000000000001010100 ; $f_{18}: 00100000100000000100000010100110$; $f_{20}: 00100000000010000100101000100100$; $f_{22}: 00101000000000000110100000100100$; $f_{24}: 00100000100000000100100010001100$; $f_{26}: 00101000000000000110000010000110$; $f_{28}: 00100000000010000100101010100100$; $f_{30}: 00100000000010000100101000101100$; $f_{32}: 00101000011001100001000000010000$ $f_{34}: 00100000000000000100100010101110$; $f_{36}: 00100000100010000100001000101100$; $f_{38}: 00101000100000000110000010100100$; $f_{40}: 00101000100000000110000000101100$; $f_{42}: 00101000000010000110001000001100$; $f_{44}: 00100000011001100001100000010000$; $f_{46}: 00100000100010000100001010101100$; $f_{48}: 00101000100000000110100010000110$ $f_{50}: 00100100110100000000100001010100$; $f_{52}: 00100000100010000100101000001110$; $f_{54}: 00100000100010000100101010000110$; $f_{56}: 00100100010100000000100001011100$; $f_{58}: 00101000011001100001000000011000$; $f_{60}: 00101000100000000110000010101100$; $f_{62}: 00101000100010000110101000000100$; 
$f_{63}: 00100000000010000100101010101100$; $f_{65}: 00100100110100000000000001011100$; $f_{67}: 00101000100010000110101000001100$; $f_{69}: 00101000100010000110101010000100$; $f_{71}: 00101000111011100001000000010000$; $f_{73}: 00101000100010000110001000100110$; $f_{75}: 00100110010101000000000011010010$; $f_{77}: 00100000111011100001100000010000$; $f_{79}: 00100000010001000101010001011100$; $f_{81}: 00101000000010000110101000100110$; $f_{83}: 00100000011001100011100000110000$; $f_{85}: 00100100010100100000000001011110$; $f_{87}: 00100110010111100000000001010000$; $f_{89}: 00101100110110000000000011010100$; $f_{91}: 00101100111100000000000001110100$; $f_{93}: 00100110110101000000000001011010$; $f_{95}: 00100100110100000000100011011100$; $f_{97}: 00100000011001100011100000111000$; $f_{99}: 00100000010001000101010011011100$; $f_{101}$ :00101100110110000000100001010100; $f_{103}: 00100110010111100000000001011000$; $f_{105}: 00100100011110000000000001111100$; $f_{107}$ :00100110010111100000100001010000; $f_{109}$ :00101000111011100001000010010000; $f_{111}$ :00100000011011100001101000010010; $f_{113}$ :00101000000010000110101000101110; $f_{115}$ : 00101000100010000110001010100110 ; $f_{117}$ :00101000011001100011000010110000; $f_{119}: 00100000111001100001101000010010$; $f_{121}$ :00101100011100000000000011110100; $f_{123}$ : 00100100011110000000000011110100 ; $f_{125}$ :00100110010101000000100011010010; $f_{127}$ :00101000011001100011000010111000; $f_{129}$ : 00100000011011100001101010010010 ; $f_{131}: 00100100110100100000100001011110$; $f_{133}$ : 00101000111011100001000010011000 ; $f_{135}: 00101110010101100000100001011000$; $f_{137}$ : 00101100110110100000000001010110 ; $f_{139}$ : 00101100010110100000000011010110 ; $f_{64}: 00100100010100000000100011010100$ $f_{66}: 00100100010100000000000011011100$; $f_{68}: 00100100110100100000000001010110$; $f_{70}: 00101110010101100000000001010000$ $f_{72}: 00101000011001100001000010011000$; $f_{74}: 00100100010100100000000011010110$; $f_{76}: 00100110110101000000000001010010$; $f_{78}: 00100000011001100001100010011000$ $f_{80}: 00100000100010000100101010001110$; $f_{82}: 00100000010001000101010011010100$; $f_{84}: 00101000100000000110100010001110$; $f_{86}: 00100100010100100000100001010110$; $f_{88}: 00100100010001100000010001001110$; $f_{90}: 00100000111011100001100010010000$; $f_{92}: 00101100110110000000000001011100$; $f_{94}: 00100000111011100001100000011000$; $f_{96}: 00100100110011100000010001000110$; $f_{98}: 00100000110011000101010001010100$; $f_{100}: 00100000011001100011100010110000$; $f_{102}: 00100110110101000000100001010010$; $f_{104}: 00101100011100000000100001110100$; $f_{106}: 00101100010110000000100001011100$; $f_{108}: 00101110010101100000000001011000$; $f_{110}: 00101000011001100011000000111000$ $f_{112}: 00101000100010000110101010001100$; $f_{114}: 00100100111110000000000001110100$; $f_{116}: 00101000111011100001000000011000$; $f_{118}: 00101000111001100001001000010010$ $f_{120}: 00100110010101000000000011011010$ $f_{122}: 00101100010110000000000011011100$; $f_{124}: 00101100010110000000100011010100$; $f_{126}: 00101110010111000000000011010010$; $f_{128}: 00100100110100100000000011011110$; $f_{130}: 00100000111001100001101010010010$; $f_{132}: 00101000100010000110001010101110$ $f_{134}: 00100100110100100000100011010110$; $f_{136}: 00101110110111000000000001010010$ $f_{138}: 00100100010100100000100011011110$; $f_{140}: 00100110111101000000000001110010$; 
$f_{141}: 00100110011101000000000011110010$; $f_{143}$ : 00101000110001000101110001010100 ; $f_{145}: 00101000111001100001001010010010$; $f_{147}$ :00100000111011100001100010011000; $f_{149}: 00100100011110100000000001110110$; $f_{151}: 00101100011100100000000001110110$; $f_{153}: 00100110011101000000000011111010$; $f_{155}$ :00101000111001100011001000110010; $f_{157}$ :00101100111100000000100011110100; $f_{159}$ : 00100110110111100000100011010000; $f_{161}$ :00100110011101000000100011110010; $f_{163}$ :00100000011011100011101000110010; $f_{165}$ :00100000111001100011101000110010; $f_{167}$ :00100110111101000000000001111010; $f_{169}$ :00101110111111000000000001110010; $f_{171}$ :00100100111110100000000011110110; $f_{173}$ :00100110111101000000100001111010; $f_{175}$ :00101100110110100000100011010110; $f_{177}$ :00101110011111000000000011110010; $f_{179}$ :00101100011100100000100011110110; $f_{181}$ :00100100011110100000100011110110; $f_{183}: 00100000111001100011101010110010$; $f_{185}$ :00101000011011100011001000111010; $f_{187}$ :00101100011100100000100001111110; $f_{189}$ : 00100100011110100000100001111110; $f_{191}$ :00101100111100100000100011111110; $f_{142}: 00101000010011000101110001010100$ $f_{144}: 00101000000010000110101010101110$; $f_{146}: 00101000011011100001001000011010$ $f_{148}: 00101100010110100000100001010110$; $f_{150}: 00101100010110100000000001011110$; $f_{152}: 00100110110111100000000011010000$; $f_{154}: 00100100111110000000100001111100$; $f_{156}: 00101110110111000000100001010010$ $f_{158}: 00101100110110000000100011011100$ $f_{160}: 00101100111100000000100001111100$ $f_{162}: 00100000111011100011100000111000$ $f_{164}: 00100000010011000101011001011110$; $f_{166}: 00101000111011100011000000111000$ $f_{168}: 00100110111101000000100001110010$ $f_{170}: 00100100111110100000000001111110$ $f_{172}: 00101100110110100000100001011110$ $f_{174}: 00101100111100100000100001110110$; $f_{176}: 00100100111110100000100001110110$ $f_{178}: 00100100011110100000000011111110$ $f_{180}: 00101100111100100000000001111110$; $f_{182}: 00101100011100100000000011111110$ $f_{184}: 00101000111001100011001010110010$ $f_{186}: 00101100110110100000000011011110$ $f_{188}: 00101100111100100000000011110110$; $f_{190}: 00101000011011100011001010111010$ $f_{192}: 00100100111110100000100011111110$.

\section{$\Phi_{5} \cap\left(\mathscr{P}_{\mathscr{B}_{5}} \backslash\left(\mathscr{L}_{5} \cup \mathscr{R}_{5}\right)\right)$, барьер длины $k=5$ :}

$f_{1}: 00001000000000000001000000010000$; $f_{3}: 00001000000000001001000010010000$; $f_{5}: 00001000100000000000000011010000$; $f_{7}: 00001000000000000001001010010010$; $f_{9}: 00001000000000000000000010111100$; $f_{11}: 00001000110100000000000011010000$; $f_{13}$ :00001000100000001000000000111100; $f_{15}$ :00001000000000000011001010110010; $f_{17}$ :00001000100000001000000001111010; $f_{19}: 00001000100000001101010001010100$; $f_{2}: 00001000010100000000000001010000$;

$f_{4}: 00001000110100000000000010000000$; $f_{6}: 00001000000000001001001000010010$; $f_{8}: 00001000100000000000000000011110$; $f_{10}: 00001000010100000010001010000000$; $f_{12}: 00001000000000001000000010011110$; $f_{14}: 00111010001100100000000010000000$; $f_{16}: 00001000000000001011001000110010$; $f_{18}: 00001000000000001000000011111010$; $f_{20}: 00001000100000000101010011010100$; 
$f_{21}: 00001000110101001000000001010100 ; \quad f_{22}: 00001000010101001000000011010100$; $f_{23}: 00001000100000000101011001010110 ; \quad f_{24}: 00001000010101100000000011010110$; $f_{25}: 00110000101110101000101000001000 ; \quad f_{26}: 00110000101110100000101010001000$; $f_{27}: 00011100100101001001010000101000 ; \quad f_{28}: 00001000110101000111110010101000$; $f_{29}: 00001000100000000111111001111110 ; \quad f_{30}: 00001000011111100000000011111110$.

\section{$\Phi_{5} \cap\left(\mathscr{P}_{\mathscr{P}_{5}} \backslash\left(\mathscr{L}_{5} \cup \mathscr{R}_{5}\right)\right)$, без барьера:}

$f_{1}: 00001000100100100000000010000000 ; \quad f_{2}: 01011000010100000000000010000000$; $f_{3}: 01011000101000000000000010000000 ; \quad f_{4}: 01011000000000000010001010000000$; $f_{5}: 00011010000001100000000010000000 ; \quad f_{6}: 01011000000001100000000010000000$; $f_{7}: 01010010010001001000000010000000 ; \quad f_{8}: 01011100100001000000000010000000$; $f_{9}: 01011100001001000000000000100000 ; \quad f_{10}: 01111000001000000000000011010000$; $f_{11}: 00010110100000000000000000011110 ; \quad f_{12}: 00111010000011100000000010000000$; $f_{13}: 00011010100000101000001010000000 ; \quad f_{14}: 00111000100010000000101010000000$; $f_{15}$ :00100101110110001000100010000000; $f_{16}: 00101011001100101000001010000000$; $f_{17}: 00011001101100101000001010000000 ; \quad f_{18}: 01011110001010000000000010101000$; $f_{19}: 01011000010100000000000011010110 ; \quad f_{20}: 01111010100000000010001011010000$; $f_{21}$ :01010010010001001000000010011110; $f_{22}: 01011100110101000000000011010000$; $f_{23}$ :01010010001010101010010010000100; $f_{24}: 00001000001111000011001010110010$; $f_{25}: 00101011001100011011000110110000 ; \quad f_{26}: 00101000000011011101110111010000$; $f_{27}: 01011110011111100000000011111110$.

Статья поступила 17.09.2008. 